\title{
Laparoscopic surgical approach for a borderline ovarian tumour of more than $25 \mathrm{~cm}$
}

\author{
S. Tamburro • E. Nohuz $\cdot$ G. Mage $\cdot$ M. Canis
}

Received: 1 March 2009/Accepted: 22 April 2009/Published online: 14 May 2009

(C) Springer-Verlag 2009

\begin{abstract}
Guidelines for surgical treatment of borderline ovarian tumours include peritoneal washing, hysterectomy with bilateral salpingo-oophorectomy, omentectomy and multiple peritoneal biopsy. Several authors indicate the laparoscopy as possible standard approach to treat women with benign ovarian tumours. We describe a 58 -year-old menopausal woman consulting for bleeding and abdominal volume; pelvic pain occurred occasionally. Magnetic resonance and ultrasound evidenced a big ovarian tumour as more than $23 \mathrm{~cm}$ in diameter with an intracystic mass of $5 \mathrm{~cm}$. Laparoscopic treatment was exhaustively performed. No surgical complication occurred and no treatment was necessary after surgery.
\end{abstract}

Keywords Large borderline - Ovarian tumour · Laparoscopy

\section{Introduction}

Borderline (low-malignant potential) ovarian tumours account for $10 \%$ to $20 \%$ of all ovarian epithelial tumours and are mainly diagnosed in young women or most usually in postmenopausal women.

Guidelines for surgical treatment of borderline ovarian tumour are similar to those for ovarian cancer and include laparotomy with peritoneal washing, hysterectomy with

S. Tamburro $(\bowtie) \cdot$ E. Nohuz

CH Thiers, Gynecology and Obstetrics,

Thiers, France

e-mail: s.tamburro@cice.fr

G. Mage $\cdot$ M. Canis

Polyclinique Hotel Dieu,

CHU Clermont Ferrand-Gynecology and Obstetrics,

Clermont Ferrand, France bilateral salpingo-oophorectomy, omentectomy, and multiple peritoneal biopsies, although several authors indicate laparoscopy as the standard approach for the treatment of women with benign ovarian tumours.

In young women with early-stage disease, conservative surgery aimed at preserving childbearing potential is associated with a recurrence rate of $0 \%$ to $30 \%$ and the overall survival is not negatively affected if recurrences are treated surgically.

Despite the use of modern diagnostic tools such as transvaginal sonography, colour Doppler and serum tumour markers, it sometimes remains difficult to distinguish between benign borderline and malignant ovarian tumours. Owing to the non-specific macroscopic aspect of borderline ovarian tumours and the relatively poor diagnostic accuracy of intra-operative histology, borderline ovarian tumours may fail to be recognised or adequately treated during laparoscopy.

We describe here a case of very large borderline ovarian cyst in a 58-year-old menopausal patient who underwent laparoscopic treatment and who as yet not presented any recurrence of disease at 3-years follow-up.

\section{Case}

A 58-year-old menopausal woman attended our consultation for abnormal uterine bleeding and abnormal increased abdominal volume associated with occasionally episodes of pelvic pain. She is $163 \mathrm{~cm}$ tall, weighs $68 \mathrm{~kg}$ and suffers from gastric ulcer and psoriasis. With respect to her obstetrics history, she had three uncomplicated pregnancies and used oral contraception for 8 years. She underwent appendectomy and tumorectomy for breast cancer followed by tamoxifen treatment 10 years ago. Five years before our 
consultation a gynaecological evaluation evidenced the presence of a $5-\mathrm{cm}$ left ovarian mass. At our examination no abdominal pain was induced by palpation but a pelvic mass around $15 \mathrm{~cm}$ in diameter was found with the superior limit close to the umbilical line.

Ultrasound examination performed by endovaginal probe revealed a large ovarian cyst, measuring over $20 \mathrm{~cm}$ and characterised by vascular and tissular structures and septa with low IR. The intracystic mass measured $3 \mathrm{~cm}$ with cloudy fluid; no papillary structures were found on the surface of the cyst and no intrabdominal free fluid was found.

No modification of haematological values for coagulation, liver function, platelets, blood cells and inflammatory proteins were detected and the markers for CA 125, CEA, CA 19-9 and CA15-3 were negative.

Magnetic resonance study with $\mathrm{T} 2$ and $\mathrm{T} 1$ fat saturation showed an ovarian multilocular mass of $22.5 \times 12.9 \times 17 \mathrm{~cm}$ (Fig. 1). Uterine section demonstrated the presence of multiple myoma nodules associated with brawny endometrium. NMR images showed that pelvic, iliac and lomboaortic lymph nodes were negative for metastatic involvement.

\section{Techniques and instrumentation}

Two weeks after the diagnosis the patient was hospitalised and laparoscopic treatment was performed. We used a sub-costal access by Verres-Palmer needle to obtain pneumoperitoneum

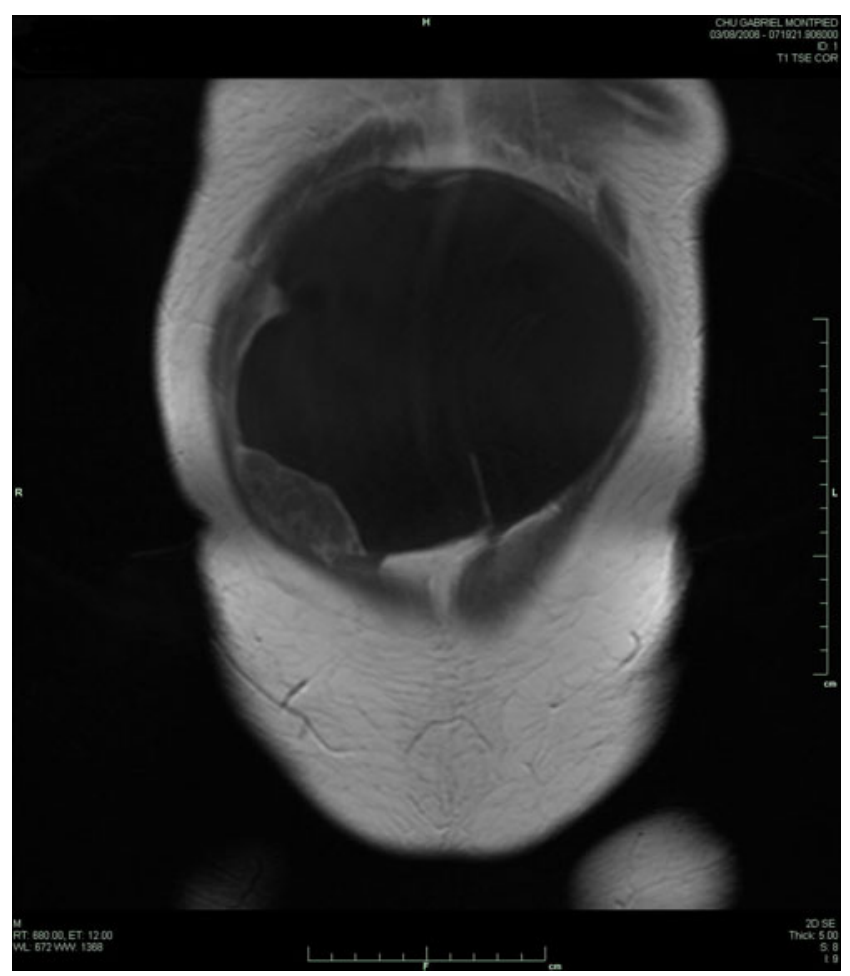

Fig. 1 Fat saturation showing an ovarian multilocular mass and a 5-mm camera was introduced to explore the abdominal cavity.

No lesions were observed in the peritoneum and there was no ascitic fluid but the ovarian tumour occupied the whole abdominal space. Three suprapubic trocars way were installed for the adnexectomy. To start with we introduced a vicryl 0 endoloop, created manually by a Roeder modified knot, through which a metallic 5-mm trocars was passed. Cystic fluid was aspirated through a $5 \mathrm{~mm}$ metallic trocars by a suction probe, while at the same time we progressively closed the endoloop within the cystic wall via the lateral trocars. Any cyst fluid spillage was verified. Intraperitoneal right adnexectomy was performed by coagulation and section of the infundibolopelvic vessels by a bipolar "Robi" device, scissors and Manhes grasping forceps (Karl Storz, Tuttlingen, Germany), according to the technique in general use in our department.

To remove the ovary in complete safety we used a large endobag (MTP) closed in a such a way as to obtain one big envelope containing the ovarian mass, which was finally extracted through the abdominal wall by a $10-\mathrm{cm}$ incision made along the previous appendectomy scar and sutured by a flexocryn 3/0. Histological examination on frozen sections concluded this was a borderline ovarian serous cancer with intracystic papillary structure of $5 \mathrm{~cm}$ diameter (Fig. 2).

During a second phase, a laparoscopic-assisted hysterectomy, adnexectomy and omentectomy were carried out.

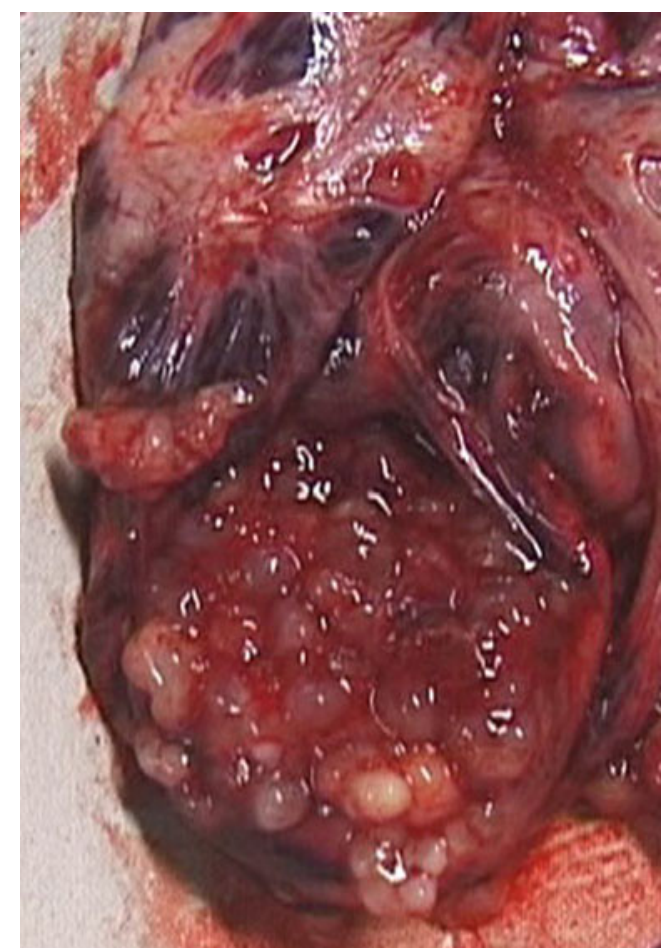

Fig. 2 Histological examination on frozen sections of a borderline ovarian serous cancer with intracystic papillary structure 
No surgical complication occurred during surgery and the operative time was $250 \mathrm{~min}$. There were no post-operative complications and only analgesic treatment was administered to the patient. After 3 days, Ms. I. A. left the hospital and returned home with simple FANS treatment. One month later at clinical gynaecological examination no metastatic lesions, no ascitic peritoneal fluid, no symptoms of pain were found and serum cancer markers were all negative. Every 6 months serum markers are tested and liver ultrasound examination, lung exploration and gynaecologic evaluation take place. This case was presented to the Oncology committee and no supplementary treatment was found to be necessary: as of today no recurrence of the pathology has been evidenced.

\section{Conclusion}

Currently laparoscopy is considered the best choice of surgical treatment for adnexal pelvic masses; however, its role in management of large borderline cyst is still questioned [1, 2]. Several authors concluded that this treatment is feasible with fewer complications and shorter hospitalisation provided the tumour size is less than 10 [3] or $5 \mathrm{~cm}$ [4], underlining also that long-term follow-up and fertility rates are similar between laparoscopy and laparotomy techniques [5]. Others show that the only difference seems to concern the post-operative complications that appear less after laparoscopy [3] than laparotomy [6, 7]. In our opinion many factors have to be considered when assessing the feasibility of the laparoscopic approach for very large ovarian tumours with low malignancy. Serum marker levels, the size and ultrasound index of resistance of cystic vegetations, the absence or presence of ascitic peritoneal fluid are important data that will be considered as the clinical limit for the laparoscopic approach. The technical limits are posed by the possibility of creating sufficient safe minimal peritoneal space by the pneumoperitoneum to expose the peritoneal surface in order to exclude suspect implants.
In our case report the size of the ovarian mass was not a limit, suggesting that laparoscopic surgical treatment for ovarian borderline tumour should be feasible and safe independently of the size of lesion. However, in accordance with recent studies [7-9], we conclude that in case of large low malignancy tumour, establishment of a surgical strategy prior to surgery is necessary to limit the possibility of contamination and to ensure safe and radical treatment.

Conflict of interest There is no actual or potential conflict of interest in relation to this article.

\section{References}

1. Malik E, Bohm W, Stoz F, Nitsch CD, Rossmanith WG (1998) Laparoscopic management of ovarian tumors. EcoHealth 12:13261333

2. Maneo A, Vignali M, Chiari S, Colombo A, Mangioni C, Landoni F (2004) Are borderline tumors of the ovary safely treated by laparoscopy? Gynecol Oncol 94:387-392

3. Odegaard E, Staff AC, Langebrekke A, Engh V, Onsrud M (2007) Surgery of borderline tumors of the ovary: retrospective comparison of short-term outcome after laparoscopy or laparotomy. Acta Obstet Gynecol Scand 86:620-626

4. Mathevet P, Nessah K, Dargent D, Mellier G (2003) Laparoscopic management of adnexal masses in pregnancy: a case series. Eur $\mathrm{J}$ Obstet Gynecol Reprod Biol 108:217-222

5. Darai E, Tulpin L, Prugnolle H, Cortez A, Dubernard G (2007) Laparoscopic restaging of borderline ovarian tumors. EcoHealth 21:2039-2043

6. Brosi N, Deckardt R (2007) Endoscopic surgery in patients with borderline tumor of the ovary: a follow-up study of thirty-five patients. J Minim Invasive Gynecol 14:606-609

7. Eltabbakh GH, Charboneau AM, Eltabbakh NG (2008) Laparoscopic surgery for large benign ovarian cysts. Gynecol Oncol 108:72-76

8. Desfeux P, Bats AS, Bensaid C, Chatellier G, Blanc B, Querleu D et al (2007) Impact of the surgical route on staging and outcome of early borderline ovarian tumors. Gynecol Obstet Fertil 35:193198

9. Romagnolo C, Gadducci A, Sartori E, Zola P, Maggino T (2006) Management of borderline ovarian tumors: results of an Italian multicenter study. Gynecol Oncol 101:255-260 